\title{
Rendezvous Technique for the Treatment of Complete Common Bile Duct Transection After Multiple Hepatobiliary Surgeries
}

\author{
Seung Shin, MD, Ariel Klevan, MD, Christopher A. Fernandez, MD,,2 \\ J. Andres Astudillo, MD, and Jose Martinez, MD ${ }^{1}$
}

\begin{abstract}
Common bile duct (CBD) injury during surgical procedures is a serious complication. Partial injury can usually be managed by a combination of percutaneous and/or endoscopic techniques. However, the management of complete transection of the CBD is very challenging. There are small case series of nonsurgical management of complete CBD transection during laparoscopic cholecystectomy. In this particular case, a 55-year-old female patient had multiple operations because of malignant pheochromocytoma with liver metastases. She developed a complete CBD transection during right hepatectomy. A biloma was managed with image-guided percutaneous drainage. However, both attempts of percutaneous transhepatic cholangiography (PTC) and endoscopic retrograde cholangiopancreatography (ERCP) for $\mathrm{CBD}$ stent were unsuccessful, as the native $\mathrm{CBD}$ was partially resected during the injury. A rendezvous procedure, in which a guidewire was placed through the distal CBD and into a biloma by ERCP and subsequently snared with PTC, allowed for a biliary-duodenal catheter to be placed successfully and achieve continuity of the patient's biliary tree.
\end{abstract}

\section{Introduction}

$\mathbf{C}$ OMPLETE TRANSECTION OF THE common bile duct (CBD) is a serious complication of hepatobiliary surgery. In laparoscopic or open cholecystectomy, the mean incidence of CBD transection is $0.31 \%$ (range, $0 \%-3.75 \%$ ). ${ }^{1,2}$ The standard treatment includes an open surgical approach with hepaticojenunostomy or choledochojejunostomy, depending on the level of injury. The rendezvous procedure, a combined endoscopic-radiologic approach, has been applied and reported since 1987. Most of the case reports or articles are of laparoscopic cholecystectomy-related injury. This procedure is mainly used to assist in gaining access to the CBD. There are two possible methods for this procedure. One is that a guidewire is placed endoscopically by endoscopic retrograde cholangiopancreatography (ERCP), and a radiologist subsequently snares the free end of the guidewire via percutaneous entry. ${ }^{3}$ The other involves the reverse order, in which the endoscopist snares the guidewire that has been placed percutaneously. Grönroos ${ }^{4}$ has reported the use of a rendezvous procedure to treat a CBD stricture and leak secondary to injury sustained during laparoscopic cholecystectomy.

In this particular case, the patient's clinical situation was complex for several reasons. First, the patient had undergone four previous operations, including two hepatectomies for malignant pheochromocytoma with liver metastases. Furthermore, the injury included a complete CBD transection and partial resection, which was not recognized in the operating room. Thus, a surgical approach in this situation would carry a significant risk of further complications to the patient and is best avoided, whenever possible.

\section{Clinical Case}

We report a case of a 55-year-old female patient with an extensive surgical history for a diagnosis of malignant pheochromocytoma. The patient originally underwent a right adrenalectomy. She subsequently developed metastatic recurrence 2 years later and underwent a resection of a left adrenal tumor, hepatic segmentectomy ( 2 and 4 ), and cholecystectomy. Within 7 months, further recurrences necessitated staged laparotomies, including a distal pancreatectomy and left retroperitoneal dissection, followed by extended right hepatectomy, diaphragm resection, lysis of adhesions, and radiofrequency ablation 12 days later. Postoperatively, the patient demonstrated an elevation of total bilirubin level up to $2 \mathrm{mg} / \mathrm{dL}$, with large amount of bilious fluid draining from the surgically placed Jackson-Pratt drain. She remained clinically stable and afebrile, with no notable jaundice or pain. Computed tomography of the abdomen showed a large fluid collection in the right upper quadrant with

\footnotetext{
${ }^{\mathrm{l}}$ Department of Surgery, University of Miami Miller School of Medicine, Miami, Florida
}

${ }^{2}$ Department of Internal Medicine, University of Michigan, Ann Arbor, Michigan. 


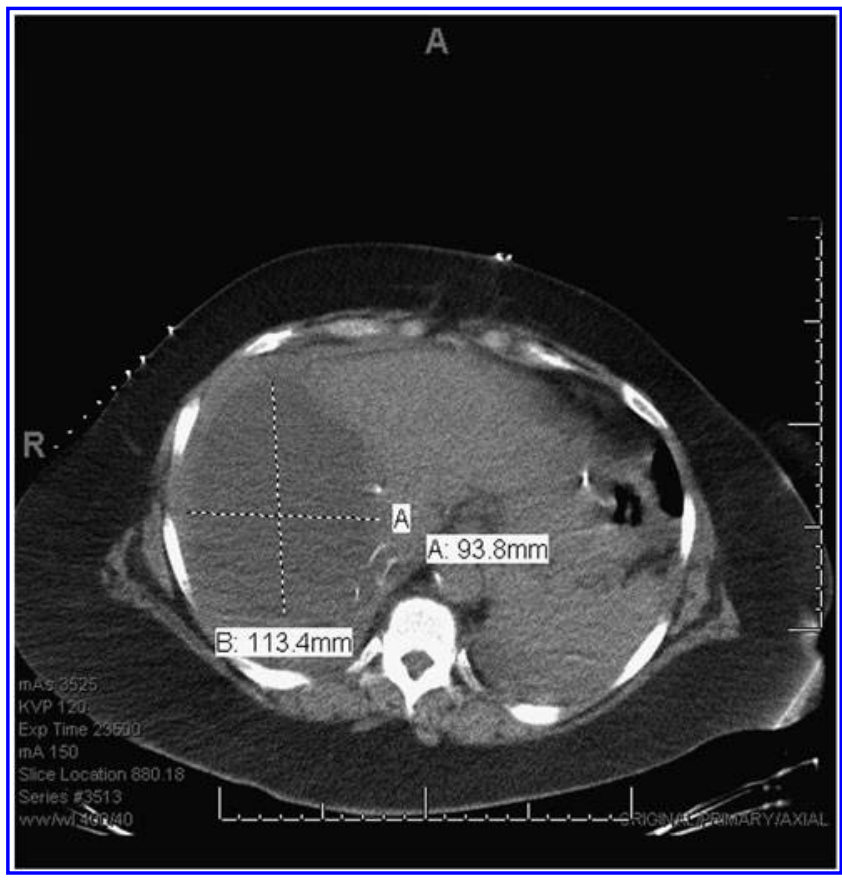

FIG. 1. Computed tomography shows a large fluid collection in the right upper quadrant with no evidence of intrahepatic biliary dilatation.

no evidence of intrahepatic biliary dilatation (Fig. 1). The collection was drained with computed tomography-guided percutaneous drainage on postoperative Day 2.

ERCP for biliary stent placement was attempted. A complete disruption of the CBD was demonstrated, and attempts at placing a guidewire into the intrahepatic biliary tree were not successful. Two days later, a percutaneous transhepatic cholangiography (PTC) attempt to place an internal-external biliary catheter was unsuccessful at recannulizing the distal

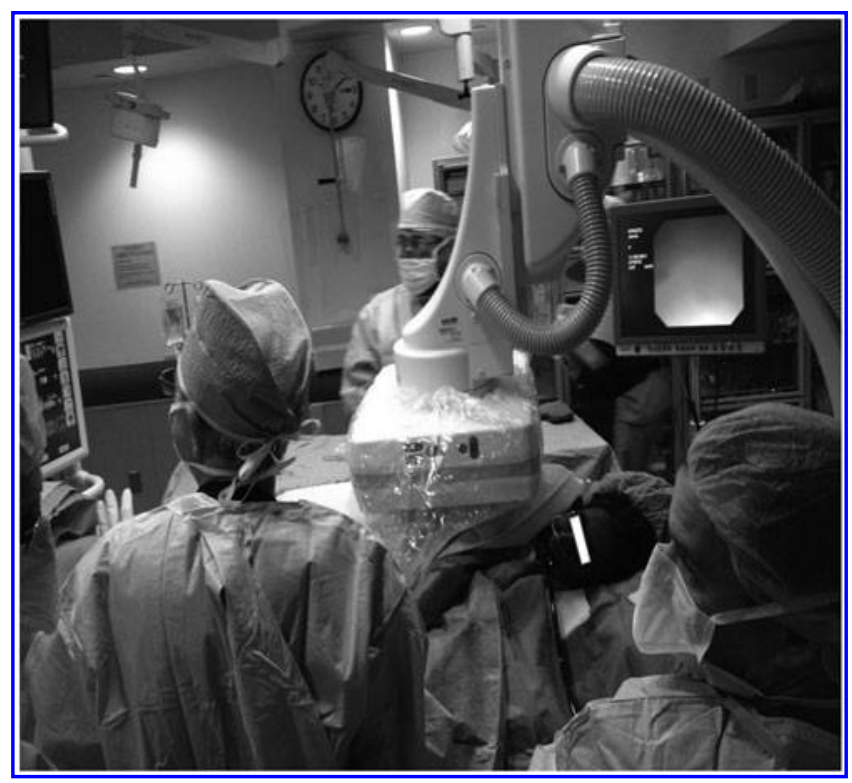

FIG. 2. Interventional radiology suite accommodating both endoscopy and interventional radiology personnel and equipment.

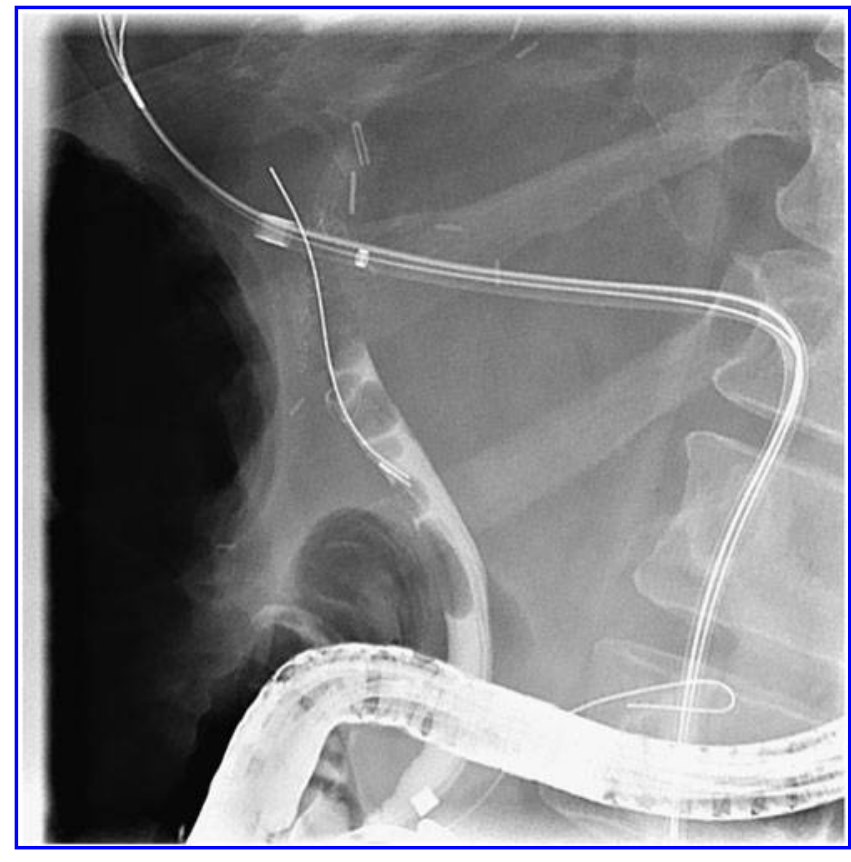

FIG. 3. Side-viewing duodenoscopy allows for cannulation of the common bile duct into the biloma.

CBD as well. A left hepatic duct catheter was placed. A segmental resection of the CBD along with its transection rendered it essentially impossible to achieve recontinuity of the biliary tree by placing a guidewire using a single method. A combined rendezvous procedure was then attempted. The procedure was set up in a large interventional radiology suite that accommodated both teams simultaneously (Fig. 2). An ERCP was carried out with a side-viewing duodenoscope, and the ampulla of Vater was cannulated, allowing for the passage of a guidewire up the distal CBD and into the biloma cavity (Fig. 3). Subsequently, PTC was performed though the patient's left-sided biliary branches into the same space. After multiple attempts, the guidewire was snared within the cavity and pulled through the existing PTC catheter outside the

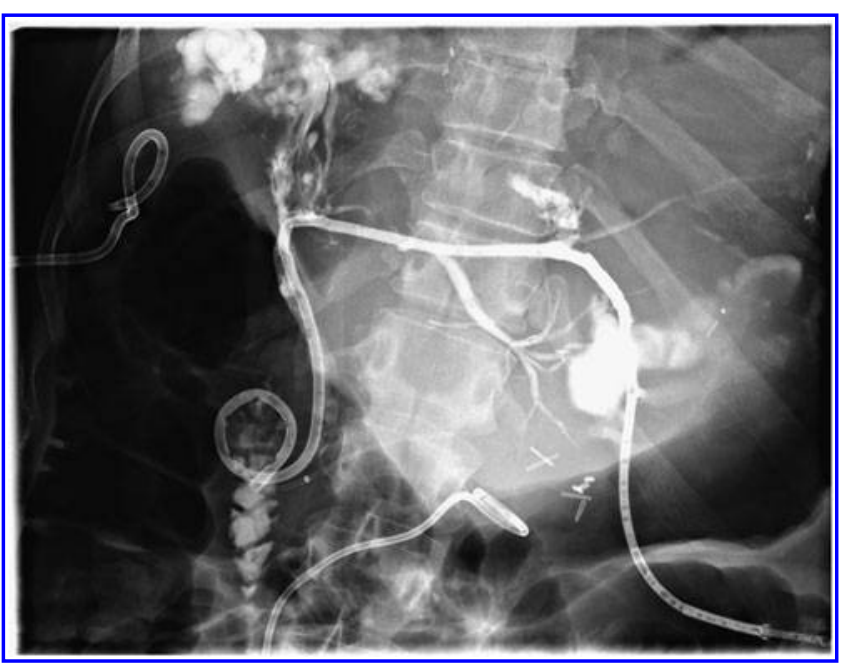

FIG. 4. Internal-external biliary-duodenal catheter achieves biliary continuity. 
abdominal wall. An internal-external biliary-duodenal catheter (8 French; Boston Scientific, Marlborough, MA) was then placed successfully, achieving biliary continuity (Fig. 4).

The patient tolerated the procedure well and was subsequently discharged home the following day in stable condition. Two months later, the patient underwent image-guided percutaneous placement of a biliary stent (WallFlex ${ }^{\mathrm{TM}} \mathrm{RX}$ biliary stent, $8 \mathrm{~mm} \times 6 \mathrm{~cm}$; Boston Scientific). This stent was subsequently removed endoscopically in 4 months. The internalexternal biliary catheter was gradually upsized up to 12 French and eventually removed 9 months after the original procedure. The biloma in the right upper quadrant was controlled with separate percutaneous drainage and was embolized by Gelfoam $^{\circledR}$ (Pfizer, New York, NY). Liver enzymes and function tests remained normal during a 9-month follow-up period.

\section{Discussion}

Although iatrogenic CBD injury is a significant complication of hepatobiliary surgery, complete transection of the common duct is even more devastating. The mean incidence of CBD injury from laparoscopic or open cholecystectomy is $0.31 \%$ (range, $0 \%-3.75 \%$ ). ${ }^{1,2}$ Statistics about the incidence of $\mathrm{CBD}$ injury from hepatectomy are lacking. Open surgical reconstruction, with a Roux-en-Y hepatico- or choledochojejunostomy, remains the standard of care to treat these injuries. ${ }^{5}$ The ideal repair is performed immediately after the injury is created, at the time of the original surgery. However, when the injury is detected postoperatively, the patient is often temporized with percutaneous drainage techniques to treat and prevent sepsis and avoid operating in an acutely inflamed field. The restoration of biliary continuity has traditionally required an operative approach, especially in injuries of complete CBD transection and segmental resection.

A minimally invasive approach to restore biliary continuity and prevent ongoing leakage is much more desirable, as it can allow for a sick patient to avoid an extensive laparotomy and reconstruction. The rendezvous procedure, first described in 1987 by Sommer et al., ${ }^{6}$ is a combined endoscopic and imageguided percutaneous procedure that allows for the passage of a guidewire and subsequent catheter from one transected biliary segment to the other, thus recreating normal anatomic and physiologic biliary flow. This technique has traditionally been used as a method to facilitate CBD cannulation. Among several prior reports about the rendezvous procedure, only a few of them were about complete transection of the main bile duct, which was caused by cholecystectomy and blunt trauma. $3,7,8$ We are not aware of any reports on the use of the procedure to repair an unrecognized, complete $\mathrm{CBD}$ transection with partial resection, obtained as a result of open hepatobiliary surgery. In this particular case, the patient's clinical situation was further complicated by the fact that she had undergone four previous significant laparotomies, including two hepatectomies for malignant pheochromocytoma with liver metastases. A surgical approach in this situation would carry a significant risk of further complications to the patient, and therefore the rendezvous procedure was the best option for management.

This case presented with several inherent challenges. The initial attempts at achieving biliary continuity with independent ERCP and PTC were unsuccessful because the CBD had sustained a partial resection along with its transection, thus completely dismembering it. The chances of "blindly" cannulating the opposing side through the biloma cavity with a single method would be exceedingly low. This type of injury necessitated the use of the combined procedure as the only option available to avoid taking the patient back to the operating room for a formal exploration. Furthermore, once the guidewire was snared percutaneously and access was achieved, an acute angle between the two segments created an additional challenge. This required the maintenance of tight control at both the oral and percutaneous ends of the wire to allow the catheter the ability to negotiate this angle and gain entry into the distal CBD and duodenum. The implementation of this procedure should be limited to centers that have the available resources and experience in both therapeutic ERCP and interventional radiology.

\section{Conclusions}

The combination of CBD transection and partial resection is a devastating iatrogenic injury that traditionally requires formal surgical exploration and reconstruction. We report a case of using a combined endoscopic-percutaneous rendezvous technique to restore biliary continuity in a minimally invasive fashion.

\section{Disclosure Statement}

No competing financial interests exist.

\section{References}

1. Archer SB, Brown DW, Smith CD, et al. Bile duct injury during laparoscopic cholecystectomy: Results of a national survey. Ann Surg 2001;234:549-559.

2. Nuzzo G, Giuliante F, Giovannini I, et al. Bile duct injury during laparoscopic cholecystectomy: Results of an Italian survey. Arch Surg 2005;140:986-992.

3. Fiocca F, Salvatori FM, Fanelli F, et al. Complete transection of the main bile duct: Minimally invasive treatment with an endoscopic-radiologic rendezvous. Gastrointest Endosc 2011;74:1393-1398.

4. Grönroos JM. Rendezvous procedure in iatrogenic bile duct injury. Surg Laparosc Endosc Percutan Tech 2007;17:186-189.

5. Lillemoe KD. Biliary injuries and strictures and sclerosing cholangitis. In: Mulholland MW, Lillemoe KD, Doherty FM, Maier RV, Simeone DM, Upchurch GR Jr, eds. Greenfield's Surgery: Scientific Principles and Practice, 5th ed. Philadelphia: Lippincott Williams \& Wilkins, 2010, pp. 981-997.

6. Sommer A, Burlefinger R, Bayerdörffer E, Ottenjann R. Internal biliary drainage in the "rendezvous" procedure. Combined transhepatic endoscopic retrograde methods [in German]. Dtsch Med Wochenschr 1987;112:747-751.

7. Misra SP, Dwivedi M. Endoscopic management of persistent biliary leakage resulting from complete transection of the bile duct at cholecystectomy. Endoscopy 2006;38:598-603.

8. Miller T, Singhal S, Neese P, Duddempudi S. Non-operative repair of a transected bile duct using an endoscopic-radiologic rendezvous procedure. J Dig Dis 2013;14:509-511.

Address correspondence to: Christopher A. Fernandez, MD Department of Internal Medicine University of Michigan Health System 3110 Taubman Center, SPC 5368 1500 East Medical Center Drive Ann Arbor, MI 48109

E-mail: fernandc@med.umich.edu 
This article has been cited by:

1. James Meek, Savannah Fletcher, Kristen Crumley, W.C. Culp, Mary Meek. 2017. Percutaneous rendezvous technique for the management of a bile duct injury. Radiology Case Reports . [Crossref] 